\title{
A compact ultra-wideband power divider with favorable selectivity using transversal filtering transformer
}

\author{
Yu Cao ${ }^{\text {a) }}$, Xiaohong Tang, and Ling Wang \\ Fundamental Science on EHF Laboratory, School of Electronic Engineering, \\ University of Electronic Science and Technology of China, \\ Chengdu 611731, P. R. China \\ a)cyeahabs@126.com
}

Abstract: A compact ultra-wideband (UWB) power divider with favorable selectivity is proposed. A transversal filtering transformer consisting of two different centrally loaded resonators with common feeding lines is introduced at input port to obtain good in-band response and high out-of-band rejection. Simple and rapid design procedure is adopted to find the key parameters. A prototype power divider is designed and fabricated to validate the predicted features. Measured results show that the power divider is featured by sharp roll-off skirts and large stopband attenuation.

Keywords: power divider, ultra-wideband, centrally loaded resonator, transversal filtering transformer, transmission zero

Classification: Microwave and millimeter wave devices, circuits, and systems

\section{References}

[1] "Revision of Part 15 of the Commission's Rules Regarding Ultra-Wideband Transmission System," FCC, Washington, DC, Tech. Rep. ET-Docket 98-153 (2002).

[2] Y. Wu and Y. Liu: Electron. Lett. 49 (2013) 1002. DOI:10.1049/el.2013.0296

[3] K. Song and Q. Xue: IEEE Microw. Wireless Compon. Lett. 20 (2010) 13. DOI:10.1109/LMWC.2009.2035951

[4] M. E. Bialkowski and A. M. Abbosh: IEEE Microw. Wireless Compon. Lett. 17 (2007) 289. DOI:10.1109/LMWC.2007.892979

[5] C. Zhuge, K. Song and Y. Fan: Microw. Opt. Technol. Lett. 54 (2012) 1028. DOI:10.1002/mop.26745

[6] S. W. Wong and L. Zhu: IEEE Microw. Wireless Compon. Lett. 18 (2008) 518. DOI:10.1109/LMWC.2008.2001009

[7] T.-S. Dang, C.-W. Kim and S.-W. Yoon: Electron. Lett. 50 (2014) 95. DOI:10. 1049/el.2013.2290

[8] L. Guo, A. Abbosh and H. Zhu: Electron. Lett. 50 (2014) 383. DOI:10.1049/el. 2013.4160

[9] H. Zhu, A. Abbosh and L. Guo: Electron. Lett. 50 (2014) 1081. DOI:10.1049/ el.2014.1214

[10] H.-H. Hu, Z.-Y. Xiao, W.-Q. He and S. Gao: Microw. Opt. Technol. Lett. 51 
(2009) 53. DOI:10.1002/mop.24014

[11] X. Y. Zhang and Q. Xue: IEEE Trans. Microw. Theory Techn. 56 (2008) 913. DOI:10.1109/TMTT.2008.919648

[12] J. Zhao, J. Wang, G. Zhang and J.-L. Li: IEEE Microw. Wireless Compon. Lett. 23 (2013) 638. DOI:10.1109/LMWC.2013.2283873

[13] M. Mirzaee and B. S. Virdee: Electron. Lett. 49 (2013) 399. DOI:10.1049/el. 2012.4203

\section{Introduction}

Power divider plays an important role in microwave circuits and systems. Due to the rapid growth of unlicensed use of ultra-wideband (UWB) for indoor and hand-held systems, a tremendous interest has been aroused on exploring various components that operate in the specified 3.1-10.6 GHz UWB range [1]. However, the tranditional power dividers such as Wilkinson and Gysel types cannot be used in UWB systems directly due to their limited bandwidth. For this reason, some variations of these tranditional power dividers are reported to meet the demand of UWB applications. For example, the multi-section Wilkinson power divider is used to broaden the bandwidth [2]. Multilayer microstrip line-slotline coupling structure is another way to realize UWB performance [3, 4]. In [5] and [6], UWB response is obtained by introducing different kinds of impedance matching networks in two output ports of the power dividers. The UWB equal or unequal power dividers with three-line coupled structure are discussed in [7], [8] and [9]. Among the UWB power dividers mentioned above, the power dividers reported in [3], [5], [6] and [7] are more attractive since they can not only divide the needed microwave signal power but also reject the unwanted frequency signal. Integrating filter function into power dividers of this kind will be beneficial to reduce circuit size so as to save cost.

In this letter, a planar UWB power divider with filtering response is proposed and analyzed. Unlike the aforementioned power dividers in [5] and [6], there is only one compact UWB transversal filtering transformer deployed in front of the input port of the conventional Wilkinson power divider. According to a proper solution of the structure in [10], two different centrally loaded resonators are used to form a transversal filtering transformer, along with the common feeding lines. A pair of transmission zeros are created at the both sides of the passband skirts. The physical mechanism of the creation of transmission zeros is explained in detail with the concept of the transversal filter, that can be regarded as a difference and deeper understanding of the proposed filtering structure compared with [10]. Hence, good frequency selectivity can be obtained. To validate the predicted feature, a prototype power divider is designed, fabricated and measured.

\section{Analysis and design of the proposed UWB power divider}

Fig. 1(a) shows the layout of the proposed UWB power divider with filtering response, among which the $50 \Omega$ input feeding line of a typical Wilkinson power divider is replaced by a filter-like impedance transformer. The proposed transformer 


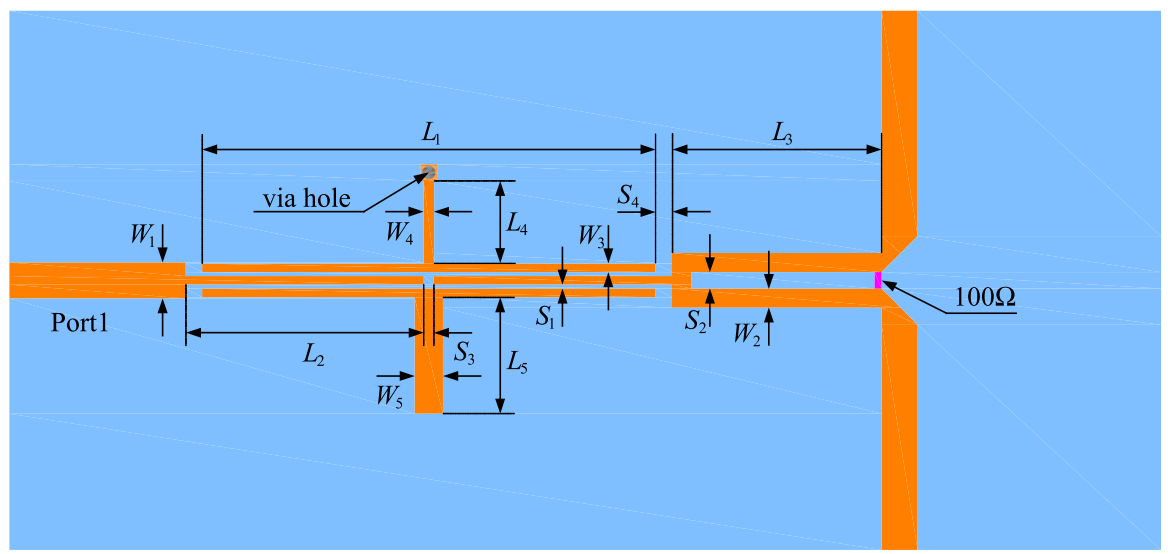

(a)

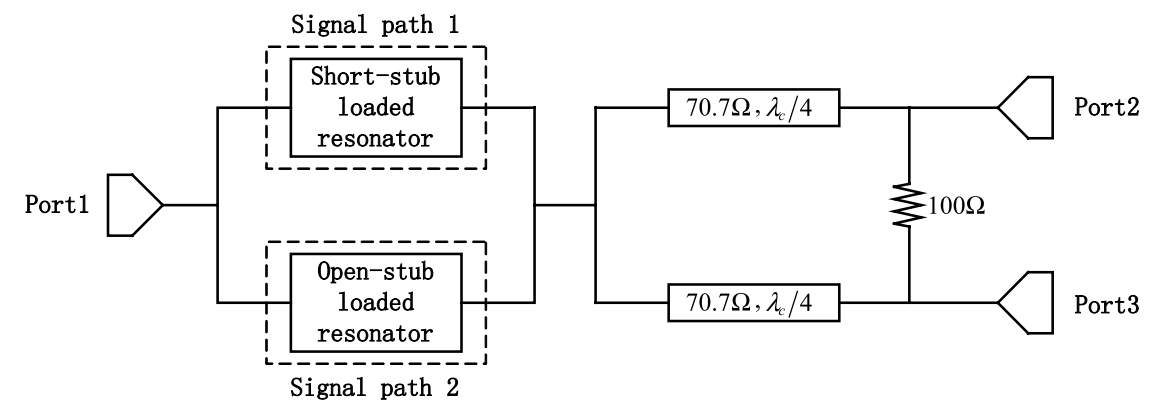

(b)

Fig. 1. Compact UWB power divider using transversal filtering transformer of (a) layout, and (b) schematic.

is composed of two centrally loaded resonators with common feeding lines. The two resonators are short- and open-stub loaded resonators, and their odd- and evenmode resonant frequencies can be expressed as $[11,12]$

$$
\begin{aligned}
f_{\text {short,odd }} & =f_{\text {open, odd }}=\frac{c}{2 L_{1} \sqrt{\varepsilon_{\text {eff }}}} \\
f_{\text {short,even }} & =\frac{c}{\left(2 L_{1}+4 L_{4}\right) \sqrt{\varepsilon_{\text {eff }}}} \\
f_{\text {short,even }} & =\frac{c}{\left(L_{1}+2 L_{5}\right) \sqrt{\varepsilon_{\text {eff }}}}
\end{aligned}
$$

where $c$ is the light speed in free space, and $\varepsilon_{\text {eff }}$ is the effective dielectric constant. Obviously, the even-mode resonant frequency of the short-stub one will be lower than that of the open-stub one. Therefore, to obtain the desired UWB response, $f_{\text {short,odd }}\left(f_{\text {open,odd }}\right)$ can be set to be center frequency $(6.85 \mathrm{GHz})$ by adjusting the length of $L_{1}$. Meanwhile, $f_{\text {short,even }}$ and $f_{\text {open,even }}$ can be set below and above $6.85 \mathrm{GHz}$, which are simply controlled by varying the length of $L_{4}$ and $L_{5}$ respectively. The symmetric tight parallel-coupled structures are formed by two resonators and their common feeding lines. They will guarantee good return loss performance within a relatively wide bandwidth. The coupling degree can be tuned by $S_{1}$ and $W_{3}$ simultaneously. The proposed transformer is normalized to $50 \Omega$, so the impedance of quarter-wave line is $70.7 \Omega$ and a $100 \Omega$ isolation resistor is used as usual. 
Fig. 1(b) depicts the schematic of the proposed power divider. Compared with the strong coupling between each one of the two resonators and the feeding line, the cross coupling between the two resonators is weak enough to be ignored. Then the impedance transformer can be seen as a transversal filter. When an UWB microwave signal passes the transformer, there might be two paths for signal transmission as shown in Fig. 1(b). When the signals pass through path 1 and path 2, and then reach at the output port, they might be equal-magnitude and out of phase [13], which might lead to the creation of transmission zeros. To study the physical mechanism of the creation of transmission zeros, the signal transmission in different coupling topologies in Fig. 2 is considered. As shown in Fig. 2, the frequency response of three kinds of transversal filters were simulated by the 3D EM simulator HFSS. The combination of a normal half-wavelength resonator and a short-stub centrally loaded resonator will generate a transmission zero in the lower stopband as shown in Fig. 2(a). Similarly, a transmission zero can also be generated in the upper stopband when a half-wavelength and an open-stub centrally loaded resonator are used together as shown in Fig. 2(b).

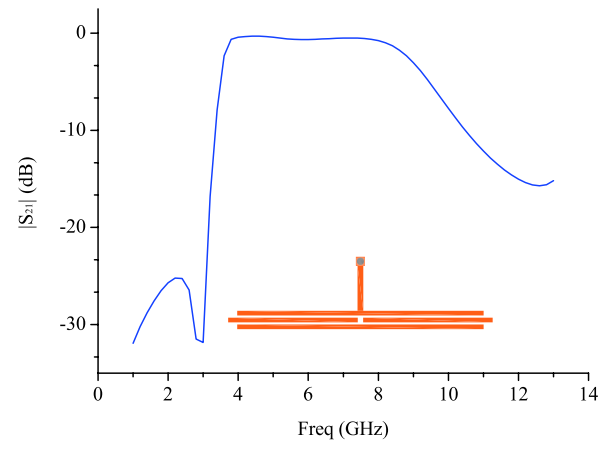

(a)

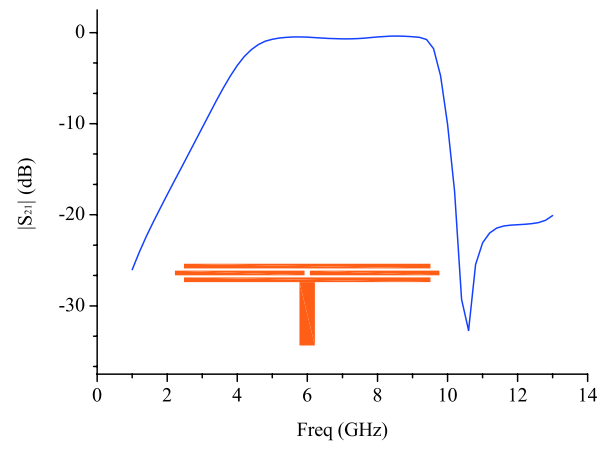

(b)

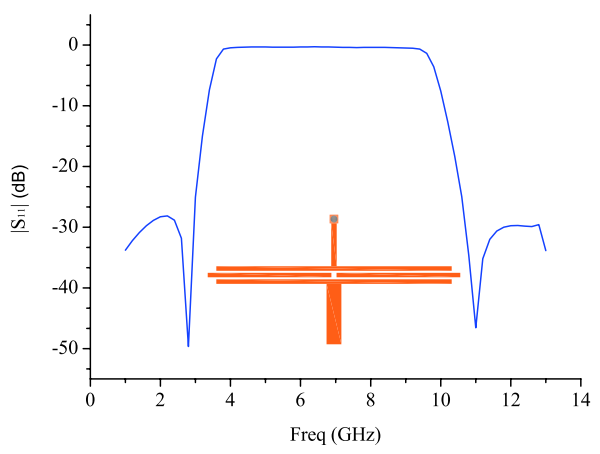

(c)

Fig. 2. The frequency responses of three kinds of transversal filters with (a) single-dual (short-stub) mode resonators, (b) singledual (open-stub) mode resonators and (c) dual-dual (open-, short-stub) mode resonators.

Fig. 2(c) shows the frequency response of the proposed transformer. The two transmission zeros are still kept at the frequency points similar as that in Fig. 2(a) and (b). It demonstrates that the filter-like impedance transformer can be analyzed through the concept of transversal filter. The roll-off skirts at the both sides of the 
passband can be improved greatly due to these two transmission zeros, then a UWB power divider with favorable selectivity can be obtained. The design procedure of the proposed power divider can be summarized as follow

1) Select the three resonant frequencies. Then find the values of $L_{1}, L_{4}$ and $L_{5}$ of the two centrally loaded resonators by the formulas (1)-(3).

2) Use the two resonators above to form the transversal filtering transformer. Find a good frequency response by tuning $S_{1}$ and $W_{3}$.

3) Design a normal Wilkinson power divider with an UWB center frequency.

4) Replace the input $50 \Omega$ transmission line of the Wilkinson power divider with the proposed transversal filtering transformer. Then tuning the size of the feeding lines and quarter-wave lines to obtain the final UWB power divider.

\section{Implementation and measurements}

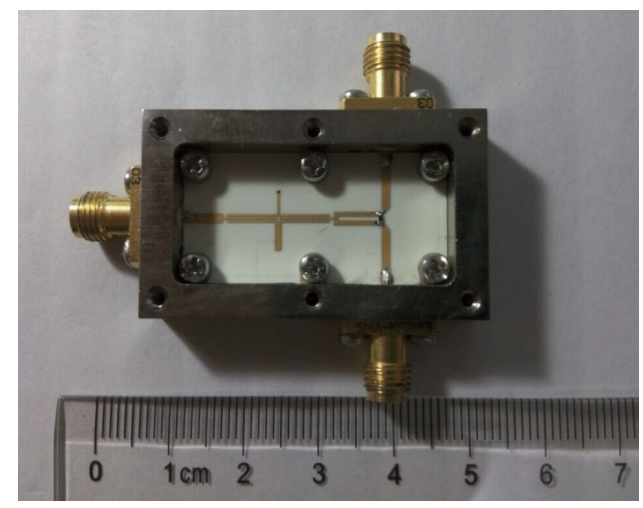

Fig. 3. Photograph of the fabricated UWB power divider

As shown in Fig. 3, a prototype divider is designed and fabricated on Rogers RO4350 substrate with a relative permittivity of 3.66, a thickness of 0.508 and a loss tangent of 0.004 . The circuit layout is simulated and optimized by using HFSS. All measured results were carried out with Agilent E8363B vector network analyzer. The final dimensions of the power divider are: $W_{1}=1.09 \mathrm{~mm}, W_{2}=0.58 \mathrm{~mm}$, $W_{3}=0.26 \mathrm{~mm}, W_{4}=0.32 \mathrm{~mm}, W_{5}=0.86 \mathrm{~mm}, L_{1}=13.7 \mathrm{~mm}, L_{2}=7.2 \mathrm{~mm}, L_{3}=$ $6.33 \mathrm{~mm}, L_{4}=2.5 \mathrm{~mm}, L_{5}=3.52 \mathrm{~mm}, S_{1}=0.12 \mathrm{~mm}, S_{2}=0.5 \mathrm{~mm}, S_{3}=0.3 \mathrm{~mm}$, $S_{4}=0.5 \mathrm{~mm}$. A $100 \Omega$ chip resistor of 0402 package is used as the needed isolation resistor. The main footprint size of the proposed power divider (without the input and output $50 \Omega$ microstrip lines) is $21 \mathrm{~mm} \times 7.55 \mathrm{~mm}$.

The measured and simulated results agree very well as shown in Fig. 4. In Fig. 4(a), $\left|S_{21}\right|$ and $\left|S_{11}\right|$ are plotted in a frequency range from 1 to $14 \mathrm{GHz}$, as well as the group delay. The measured $\left|S_{11}\right|$ in the passband is lower than $-10 \mathrm{~dB}$ and $\left|S_{21}\right|$ is about $-3 \mathrm{~dB}$ around over the frequency range of 3.87 to $9.86 \mathrm{GHz}$. The insertion loss (without consideration of the $3 \mathrm{~dB}$ power division) is less than $1 \mathrm{~dB}$ from $3.9 \mathrm{GHz}$ to $7.93 \mathrm{GHz}$, and less than $2 \mathrm{~dB}$ from $7.93 \mathrm{GHz}$ to $9.57 \mathrm{GHz}$. Due to the transmission zeros at the both sides of the passband, roll-off skirt is improved greatly. The out-of-band rejection levels are more than $30 \mathrm{~dB}$ in the lower stopband 
group delays with variation less than $0.1 \mathrm{~ns}$ from $4.5 \mathrm{GHz}$ to $9.3 \mathrm{GHz}$ show good linearity within the most part of the passband.

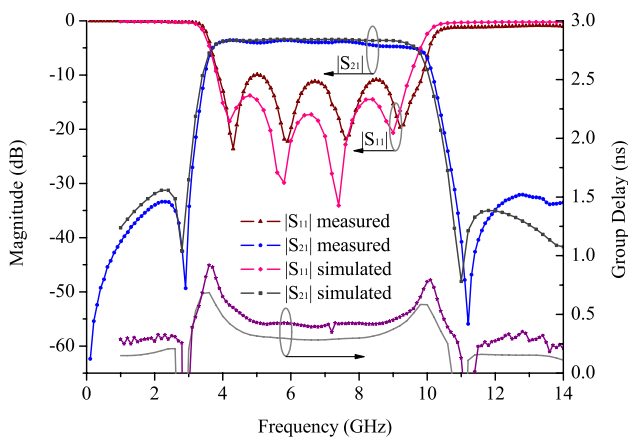

(a)

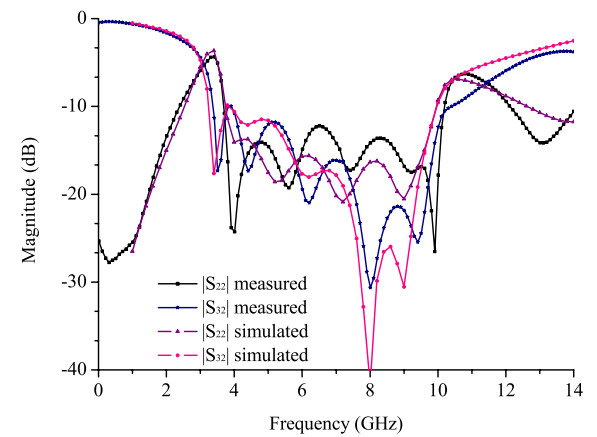

(b)

Fig. 4. Simulated and measured results of the proposed power divider: (a) magnitudes of $S_{21}$ and $S_{11}$, as well as group delay, (b) magnitudes of $S_{22}$ and $S_{32}$.

Fig. 4(b) shows the measured and simulated the magnitudes of $S_{22}$ and $S_{32}$. The measured $\left|S_{22}\right|$ of lower than $-10 \mathrm{~dB}$ from $3.7 \mathrm{GHz}$ to $10.2 \mathrm{GHz}$ proves that the proposed power divider can be a good UWB power combiner as well. The measured isolation between the two outputs is larger than $10 \mathrm{~dB}$ from $3.38 \mathrm{GHz}$ to $10.35 \mathrm{GHz}$.

Table I. Comparison of UWB power dividers with filtering response

\begin{tabular}{|c|c|c|c|c|c|}
\hline Refs. & Techniques & $\begin{array}{c}\text { Bandwidth } \\
\left(\mathrm{S}_{11}<-10 \mathrm{~dB}\right) \\
(\mathrm{GHz})\end{array}$ & $\begin{array}{c}\text { Isolation } \\
\left(\mathrm{S}_{32}<-10 \mathrm{~dB}\right) \\
(\mathrm{GHz})\end{array}$ & $\begin{array}{c}\text { Out-of-band } \\
\text { Reject } \\
(\mathrm{dB})\end{array}$ & $\begin{array}{c}\text { Circuit size } \\
\left(\lambda_{\mathrm{g}} \times \lambda_{\mathrm{g}}\right)\end{array}$ \\
\hline$[3]$ & $\begin{array}{c}\text { multilayer microstrip } \\
\text { line-slotline coupling }\end{array}$ & $3.1-10$ & $3.1-10.6$ & $\begin{array}{c}>17 \\
(0-2.3 \mathrm{GHz}) \\
(11.5-15 \mathrm{GHz})\end{array}$ & $1 \times 0.5$ \\
\hline$[5]$ & signal interference & $3.6-9.8$ & $3.1-10.6$ & $\begin{array}{c}>20 \\
(0-2.2 \mathrm{GHz}) \\
(12.2-17 \mathrm{GHz})\end{array}$ & $0.46 \times 0.72$ \\
\hline$[6]$ & $\begin{array}{c}\text { stepped-impedance } \\
\text { open-circuited stubs } \\
\text { and parallel coupled } \\
\text { lines }\end{array}$ & $3.5-10.1$ & $3.1-10.6$ & $\begin{array}{c}>18 \\
(0-2.9 \mathrm{GHz}) \\
>10\end{array}$ & $0.48 \times 1.17$ \\
\hline$[7]$ & $\begin{array}{c}\text { three parallel-coupled } \\
\text { lines with one } \\
\text { short-circuited shunt } \\
\text { stub }\end{array}$ & $3.1-10.6$ & $3.1-10.6$ & $\begin{array}{c}>10 \\
(0-2.3 \mathrm{GHz}) \\
(11.2-12 \mathrm{GHz})\end{array}$ & $0.25 \times 0.18$ \\
\hline $\begin{array}{c}\text { This } \\
\text { work }\end{array}$ & $\begin{array}{c}\text { UWB transversal } \\
\text { filtering transformer }\end{array}$ & $3.87-9.86$ & $3.38-10.35$ & $\begin{array}{c}>30 \\
(0-3 \mathrm{GHz}) \\
(10.8-14 \mathrm{GHz})\end{array}$ & $0.81 \times 0.29$ \\
\hline
\end{tabular}

Table I summarizes the main technical features of recently published UWB power dividers with filtering response, where $\lambda_{\mathrm{g}}$ represents the guided wavelength at the center frequency of UWB passband $(6.85 \mathrm{GHz})$. Obviously, the proposed UWB power divider exhibits the best out-of-band reject and second smallest circuit 
size, although some bandwidths of return loss and isolation are sacrificed. Besides, the roll-off factors of the proposed power divider are more than $30 \mathrm{~dB} / \mathrm{GHz}$ in both sidebands, which means it has stronger ability to filter the interference signals near the passband.

\section{Conclusion}

A compact UWB power divider is designed, fabricated and measured. By using the transversal filtering transformer with two different centrally loaded resonators, the proposed power divider has good frequency selectivity performance. A brief and efficient design procedure is introduced. Measurements show good agreement with the simulations. The proposed power divider is a single-sided and single-layer circuit, which means it is cheap and convenient to be fabricated and applied. 